of

\title{
INTRAVENOUS ANAESTHESIA
}

\author{
with
}

\section{TEN YEARS' EXPERIENCE IN THE USE OF PENTOTHAL SODIUM}

\author{
By \\ Ronald Jarman, D.S.C., M.R.C.s., D.A. \\ Senior Anaesthetist Princess Beatrice Hospital; Anaesthetist Royal Cancer Hospital.
}

In the year 1665 Sigismund Elsholtz first attempted intravenous anaesthesia by injecting a solution of opiate to obtain insensibility. It was not until I872 that Ore, Myer, and Witzel experimented with chloral hydrate on animals, but this method was soon abandoned. The real work on intravenous anaesthesia started about 1905, when Fedorow, St. Petersburg, reported his results on 530 cases in which he used 0.75 per cent of Hedonal $\left(\mathrm{CH}_{3} \mathrm{CH}_{2} \mathrm{CH}_{2} \mathrm{CO}\left(\mathrm{CH}_{3}\right) \mathrm{OCH} \mathrm{NH}\right)_{2}$ in a normal saline solution, and claimed that he had no fatalities.

Burkhart, after many experiments on dogs, published the results of his first forty-six cases of general anaesthesia using ether and chloroform. He injected a solution of 5 per cent to 7 per cent warmed to $28^{\circ} \mathrm{C}$. through a cannula into the vein in the arm, and found that very variable quantities, even up to several litres, were required.

Some of the patients showed syncope, cyanosis, and pulmonary oedema. The difficulties of administration were so great that Isopral $\left(\mathrm{CCL}_{3} \mathrm{CH}(\mathrm{OH}) \mathrm{CH}_{3}\right)$ was used by his successors. Burkhart, trying out a solution of a mixture of ether and isopral, reported excellent results in seventy cases.

It was during this time that Bier studied regional intravenous anaesthesia of the limbs. A variable amount of 0.5 per cent novocaine was injected into the veins near the site of the operation. - The blood supply at each end of the limb was occluded to keep the novocaine in the veins. In five to ten minutes anaesthesia was complete. From 25 to 200 c.c. of novocaine without adrenalin were required, according to the size of the limb. This method was most successful for operations on the arm, particularly the forearm.

The technique used by Gwathmey of America, when he injected 5 per cent to 7.5 per cent solution of ether in Ringer's solution was as follows:-

Thirty minutes before the operation a hypodermic injection of morphine sulphate gr. $\frac{1}{6}$ th, atropine sulphate gr. $\frac{1}{100}$ th, scopolamine gr. $\frac{1}{100}$ th was given. The solution of ether with filtered sterile Ringer's solution at a temperature of $85^{\circ} \mathrm{F}$. was thoroughly mixed in a reservoir placed eight feet above the floor. A superficial vein which was away from the operator had to be used, for the infusion, and if the elbow was selected, a padded splint was passed beneath the patient and the wrist bound to it. The skin over the chosen vein was sterilised and, under local anaesthesia, a small incision was made and the vein lifted out. The vein was then cut and the cannula inserted and tied in place. The fluid was allowed to flow through an indicator drip chamber down through rubber tubing into a blunt cannula until the usualo signs of anaesthesia appeared. When the desired degree of narcosis was obtained, the flow, which had to be continuous, was reduced by means of the control cock below the indicator. Great care was necessary to maintain an unobstructed airway. The dressings were applied before the flow was actually stopped, as the return to consciousness was often rapid. About I,000 c.c. of the solution were used per hour, and at the end of the operation the cannula was withdrawn and the wound closed. Very little solution was necessary to maintain the anaesthetic tension, once the right level of narcosis had been reached. Anaesthesia was very rapid, as the agent was introduced directly into the blood, and the degree of narcosis could be maintained exactly.

In I9I4 experiments on animals were being carried out with hedonal and ether introduced in I9Io by Siderenko (Cronstadt) and with isopral and ether and paraldehyde elsewhere, and these drugs were tried in combination with Ringer's solution. Care was taken in the analysis of the blood, urine, and faeces, but owing to the uncertainty of the various agents used, this form of narcosis did not become popular.

It was not until I920, after the Great War, that Dutrech tried ether serum and combined it with isopral, as recommended by Burkhardt and Hummell. In Mexico they went so far as to inject 
pure ethyl alcohol, but with mixed success, and an extended trial of this method in France gave bad results.

In I92I the real period of advance in intravenous anaesthesia began when Daniel and Gabriel Bardet published a paper on their results using somnifaine. At about the same time, soneryl $\left(\mathrm{C}_{4} \mathrm{H}_{9} \cdot \mathrm{C}_{2} \mathrm{H}_{5}\right.$, C . CONH . CO .) was being used by Tiffineau and de Carnot. It was Fredet and Perlis who realised that somnifaine alone was not sufficient, but found that in combination with a subcutaneous injection of morphine gr. $\frac{1}{6}$ th and scopolamine gr. $\frac{1}{100}$ th, the anaesthesia was more profound and minor operations could be attempted. The one severe drawback to these two drugs was that the patient had a very deep and prolonged sleep, and on waking complained of severe headache and drowsiness.

From that time on various chemical firms were experimenting with these barbiturates, such as pernocton and amytal. Then from America there came a full report by Zerfas, McCullum, Scholne, Swanson, Scott, and Clowes. This included two years' experimental studies on animals and the first attempts at anaesthesia on the human being by using:-

Sodium amytal $\left(\mathrm{CH}_{3}\right)_{2} \mathrm{CH}$. $\left(\mathrm{CH}_{2}\right)_{2} \cdot \mathrm{C}_{2} \mathrm{H}_{5} \cdot \mathrm{C}$. $\mathrm{CO}$. N . Na . CONH . CO) and pentobarbital sodium (nembutal) $\left(\mathrm{CH}_{3} \cdot \mathrm{CH}_{2} \cdot \mathrm{CH}_{2} \cdot \mathrm{CH} \cdot \mathrm{CH}_{3} \cdot \mathrm{C}\right.$. $\left(\mathrm{C}_{2} \mathrm{H}_{5}\right) \cdot \mathrm{CO} . \mathrm{N}$. Na . CO. NH.CO.). These were the first barbiturates to be used intravenously in a large number of cases. Owing to the long post-operative sleep and restlessness, they have been largely abandoned, but are still used for convulsions and maniacal patients. It was shown in later reports that administration by the mouth achieved better results.

In I929, Kirschner injected a 3 per cent solution of avertin $\left(\mathrm{CBr}_{3} \mathrm{CH}_{2} \mathrm{OH}\right)$ in normal saline giving I c.c. per kilo of body weight and 60 to 80 c.c. in forty-five seconds. The patient went to sleep in about one minute, and woke up five to ten minutes later, unless the narcosis was prolonged by some other form of anaesthesia.

Soneryl returned to popularity in I932. In the same year, with the introduction of sodium evipan (CO . Na.CO : N $\left.\left(\mathrm{CH}_{3}\right) \mathrm{CO} . \mathrm{C}-\left(\mathrm{CH}_{3}\right) \mathrm{C}_{6} \mathrm{H}_{9}\right)$ the former barbiturates were discontinued. Evipan was discovered by the chemists Kropp and Taub, and used first intravenously on man by Ernst Reinhoff. The patient went to sleep in a few seconds, and after a very short time regained consciousness, provided that only a small dose of the drug was used.

This drug produced suitable anaesthesia for minor operations. Other users tried it out in conjunction with nitrous oxide, oxygen, and with open ether, and found it to be a great success. Thus it naturally became the intravenous anaesthetic of
choice.

In conjunction with Abel (I933), the first paper 3 was published on Evipan. The Anaesthetic Re- $\stackrel{\Phi}{\varrho}$ search Council in this country furnished a brief $c$ report in the medical journals. Other reports $\overrightarrow{\vec{F}}$ followed, and during. I935 I was invited over to ? the United States to demonstrate the use of Evipan, and also to show a film illustrating my technique. During that short visit information $\frac{\vec{\sigma}}{\vec{\sigma}}$ was given to me that another salt or salts was being $\propto$ produced there, which, according to research is workers, was in advance of the German drug. $\overrightarrow{0}$ Through the kindness of Lundy (I935), who was doing the original work at the Mayo clinic, I was $\vec{\omega}$ given facilities for the early use of this new drug at $\frac{\Omega}{8}$ my various hospitals in London.

As Magill had introduced nembutal four or five years previously, I suggested that he should also N investigate this new drug in conjunction with ज Lundy. He declined, but advised me to proceed on similar lines as I had done with evipan. The success of both these drugs was entirely due to the $\vec{\circ}$ co-operation of the surgeons with whom I worked.

Pentothal (Jarman and Abel, I936) was released $\vec{\circ}$ in this country in r936.

Originally two drugs had been produced by re-응 search workers in America. They were knowh there as barbiturate " $A$ " and barbiturate "B," b\&t $\vec{\oplus}$ to avoid confusion they were given numbers and "A" was 8064 and "B" 8076 . Equal samples \&f " these two drugs, which were very closely allied, were sent to Lundy and myself. In the early days there was a considerable difficulty in keeping theō drugs stable as no sterile water was supplied with\%ّ them, and this was thought to be the cause. With the co-operation of the chemists this was overcome, $\overrightarrow{\overrightarrow{0}}$ and finally Lundy decided that the drug " $A$ " or 3 8064 was the one of choice, and it became known? as:-

Pentothal Sodium $\left(\mathrm{CH}_{3} \mathrm{CH}_{2} \mathrm{CH}_{2} \mathrm{CH} \quad \mathrm{CH}_{3}\right.$ 을 $\left.\mathrm{C}\left(\mathrm{C}_{2} \mathrm{H}_{5}\right) \mathrm{CO} \mathrm{N} \mathrm{Na} \mathrm{CO} \mathrm{NH} \mathrm{CS}\right)$.

Formula.-Sodium Ethyl (I - Methyl Butyl) Thio Barbituric Acid.

Description.-This is a lemon-coloured powder, bitter in taste, and smelling slightly of sulphur. It $₹$ is rapidly soluble in water, and strongly alkaline.을 The $\mathrm{pH}$ of a ro per cent solution is $10 \cdot 6$. The rapid dissociation of pentothal in the blood stream is entirely due to the sulphur atom.

Equipment.-A Vann's syringe (Io c.c.) with an eccentric nozzle, and needle size I4 with a short ${ }_{0}$ bevel, should be used in this form of anaesthesia N Only intermittent doses are given with this syringe.o For continuous intravenous anaesthesia, the appar-o atus which Abel and I designed is suggested. This is extremely compact, can be carried easily, and? may be used for other purposes. The apparatust 
consists of a Io c.c. syringe, with a three-way nozzle which is fixed to the forearm with elastic bracelets. With the control in the central position, the barrel of the syringe communicates directly via the needle to the vein. When the control is turned to the left, one lateral feed communicates directly with the needle, and this position is used for continuous sterile water-glucose infusion. When the control is turned to the right, the other lateral feed communicates with the flask containing pentothal, and the syringe can thus be loaded with a further dose of anaesthetic, which may then be injected through the needle by turning the control to the midposition.

In serious cases the patient can have a blood transfusion during the operation with pauses for anaesthetic administration. Similarly in an emergency, the syringe can be used for giving stimulants, such as coramine or alpha-lobeline; and here an advantage of the apparatus is that only a minimal amount of the drug need be given, since it is accurately measured and reaches the vein direct. We have found the administration of continuous sterile water-glucose infusion during operation of the utmost importance in some of the "poor risk" cases. Dehydration is combated and shock is largely abolished.

As this form of anaesthesia has become more and more the method of choice, so the various techniques and apparatus have multiplied.

Type of Patient.-From the point of view of anaesthesia patients may be classed as placid and resistant types of out-patients and in-patients.

The placid type will take any form of anaesthetic, not necessarily intravenous, equally well and the amount of the drug used varies only slightly; with the resistant type, however, the dosage varies considerably, so that no definite amount can be laid down as a guide to future anaesthetists. No two anaesthetics are ever the same.

Out-patients need never have any form of premedication, though it is now considered wise to give atropine just before the administration of an intravenous anaesthetic, in order to make sure that the respiratory tract is dried up. Either $\frac{1}{2} \mathrm{gm}$. or I gm. of pentothal may be administered in the surgery or out-patients' theatre, and minor operations such as removal of nails, opening of abscesses, setting of fractures, extraction of teeth, or cystoscopies, can be performed. After allowing the patient to recover in one or two hours and in some cases even less, he may go home.

The in-patient is admitted into hospital or a nursing home, or may be in a private house, and is given drugs the night before such as medinal, gr. Io, aspirin gr. Io (average adult), to ensure a good night's rest. Then, one hour before the operation, he is given an ampoule of omnopon, gr. $\frac{1}{3}$, scopolamine, gr. $\frac{1}{150}$ th. The room is darkened and the patient is left alone. In a general ward a screen is put in front of the bed and the eyes lightly bandaged. Then for the comfort of the patient $\frac{1}{2} \mathrm{gm}$. of pentothal is dissolved in Io c.c. of sterile distilled water and is injected into a vein whilst he is in bed, making sure that his airway is kept clear by taking the precaution of asking him to bite on a dental prop. He falls asleep and is taken to the theatre and placed on the operating table, where he can be given more pentothal or another anaesthetic.

The Condition of the Patient.-The usual modern methods of investigation and preparation of all patients are carried out before operation in addition to a general clinical examination and a blood and urine analysis. Special attention is paid to the blood pressure; no enema, purging or other form of dehydration is permitted for at least fortyeight hours before a major operation. The patient is supplied with plenty of fluids and glucose, and everything is done to ensure that his general condition is as good as possible.

Contra-Indications.-Pentothal is metabolised in the blood stream and not wholly in the liver as some authors have stated; breakdown is very rapid. Any gross hepatic disease or the presence of jaundice should, however, be seriously considered.

Low blood pressure.-Extreme care should be taken with any patient who shows general feebleness and low blood pressure as pentothal causes ac slight though temporary fall in blood pressure.

Posture.-When the patient is under the influence of pentothal the upright position, except, perhaps, in young healthy subjects, is contra-indicated, but under certain circumstances this can be overlooked, as, for example, when giving a spinal anaesthetic (Etherington Wilson's technique). For general routine work it is thought advisable that the recumbent posture should be adopted and even for dental work the patient should be put in an inclined plane. This is a safety measure as so many would-be anaesthetists are apt to give the drug too quickly and to administer too large a dose. If an emergency should arise, the recumbent posture is one in which the patient can be easily manipulated.

Space.-Lack of available space applies only to institutions where large numbers of patients are to be dealt with, and where facilities for recovery are inadequate.

\section{Other Barbiturates}

It was felt at first, that while this drug was being established users should be advised not to give other barbiturates prior to the injection of pentothal. This still holds good where the patient has had a long course of barbiturates, especially in the 
type, chiefly women, who complain of being unable to sleep well, and resort to such drugs as allonal, medinal and soneryl. It is an accepted practice today that nembutal may be given the night before and also one hour before the operation. The suggested dose for an adult is nembutal gr. $I_{2}^{\frac{1}{2}}$ last thing at night, and nembutal gr. 3 sixty minutes before the operation. This can be followed with atropin gr. $\frac{1}{100}$ th in order to dry up the respiratory tract.

When it is known that the patient has resorted to barbiturates as a means of obtaining sleep over a period of time, considerable care must be taken if an intravenous barbiturate is being used, because the depression of the respiratory centre in these cases is sometimes severe, and causes great anxiety.

\section{Technique and Administration}

Before going into the details of technique and administration, it is hoped that in future the teaching of intravenous therapy will take a prominent place in the student's curriculum. It is essential that the anaesthetist should have a steady hand, a knowledge of veins and be able to combat failures and minor accidents. The number of unsuccessful attempts made by would-be intravenous therapists are entirely due to lack of knowledge and experience.

Pentothal is supplied in $\frac{1}{2} \mathrm{gm}$. and i gm. ampoules. It has been proved without a shadow of doubt that the 5 per cent solution is the one of choice, though users in the United States of America prefer $2 \frac{1}{2}$ per cent or 2 per cent. This has only a small following in this country. To make a 5 per cent solution the $\frac{1}{2} \mathrm{gm}$. size has an ampoule of Io c.c. of chemically pure sterile distilled water supplied with it, and the $\mathrm{I} \mathrm{gm}$. size an ampoule of 20 c.c. These are put in a bowl of spirit together with the syringes and needles. On the table there is a little bottle of picrotoxin containing 20 c.c. $(0.3$ per cent solution) and a box of coramine containing 5 c.c. ampoules in case of accidents. The picrotoxin is used intravenously in various quantities from 2 to 5 c.c. to restore the patient to consciousness, and has been used of late with excellent results in severe barbiturate poisoning. Coramine, in case of trouble, should be given in 5 c.c. doses, either intravenously, intramuscularly or subcutaneously. A dose of this size should only be used in extreme emergency.

By the side of the table with the syringes and the ampoules there should be an apparatus by which nitrous oxide. and oxygen can be administered, such as a McKesson or Mushin. There should also be a suction apparatus.

These precautions should be applied to all types of anaesthetics, but these points are stressed since some authors are of opinion that intravenous anaesthesia is different.

A ro c.c. syringe is used because it is easier to 3 manipulate, though a 20 c.c. syringe can be used ${ }^{0}$ if preferred. The anaesthetist equips himself with a dental prop, tongue forceps and an airway, anc takes these with the syringe. Swabs and spirit ares already with the patient.

For minor operations, i.e. the setting of a wrist ote Colles's fracture, the patient is first asked to emptyo his bladder, ensuring that at least three or four hours have elapsed since his last meal; it is then sugs gested that he should lie down on a table in thes out-patient department. All tight clothing is eased, and as soon as the surgeon is ready the upperw arm is constricted by the nurse with one hand항 while she gently supports the chin with the othero The forearm is prepared with spirit, and whilst the patient is asked to clench his fist the anaesthetisto rubs the veins towards the shoulder, causing them to stand out. Selecting a suitable vein the needle is inserted with the bevel up, into the skin first, and then into the vein, thus making a valvular openingo Within fifteen seconds 3 or 4 c.c. is injected, and during this time the patient is asked to count; it is noticed that by the time he manages to reache twenty he is falling asleep. After a pause of thirty seconds a further 2 or 3 c.c. is given, aftere which the operation may be begun. If thereoiso

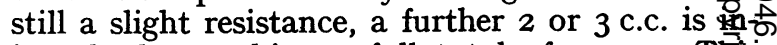
jected, thus making a full total of ro c.c. T商i dose usually suffices. On waking up during the course of the next few minutes, the patient is entirely oblivious as to what has happened. Heed rests for half an hour or so and is then allowed tơ return home.

The beginner should give only 3 or 4 c.c. in $\overrightarrow{\overrightarrow{0}}$ period of thirty seconds and repeat the next 3 or 4 c.c. at the same pace. The more advanced student can give the initial dose in fifteen to twenty? seconds, but this rate should not be exceeded. Iñ out-patients, where the patient is extremely: robust, the full ro c.c. can be given in a matter of a minute, and only allowing for the original pause of fifteen to thirty seconds after the first 4 c.c. Tof give an intravenous drug rapidly is courting danger, no matter how experienced the anaesthetiss may be or how short the operation. This mustr never be forgotten.

The disadvantages of a quick injection are that the patient complains of a severe headache, which fortunately is only temporary, double vision and $\mathrm{a}_{\mathrm{N}}$ feeling of dizziness. With regard to the slowe method, the patient is likely to recover more slowly? has a slight degree of double vision, but does not complain of other symptoms. In other words, the slow method is much more comfortable for the $e^{+}$ patient. 
With every out-patient it is always advisable to see that the stomach is emptied. I have experienced extreme anxiety with some patients who; on being admitted, have said that they have had nothing to eat or drink; after the pentothal has been given, owing to the then complete relaxation of the cardiac sphincter, large quantities of liquid have overflowed.

For in-patients the intermittent dose technique is as described above, except for the injection of omnopon gr. $\frac{1}{3}$ and scopolamine gr. $\frac{1}{150}$ th, which is given one hour previous to the operation. This technique is of considerable value as long as the veins are easy to find. In fact, it is the common practice today to give intermittent injections combined with nitrous oxide and oxygen for all straightforward operations such as appendicectomy, herniotomy, radical amputation of the breast, thyroidectomy, prostatectomy, etc.

I described this technique when using evipan in I934. It was found that by administering nitrous oxide and oxygen the anaesthetic was lengthened for short operations with the maximum amount of comfort for the surgeon; this assisted students who had difficulty in finding a suitable vein in the middle of an operation. If an apparatus like the McKesson is not at hand a gentle feed from an oxygen cylinder to the mouth adds safety. In this type of case, to save disturbing the surgeon, an arm should be left out, supported by the nurse in charge, to enable the anaesthetist to give further injections when necessary. For all short operations such as cystoscopies, sigmoidoscopies, laryngoscopies, bronchoscopies, etc., there is very little need ever to use more than $\frac{1}{2} \mathrm{gm}$. or $\mathrm{I} \mathrm{gm}$. of penthothal.

The continuous technique is somewhat different. With the advent of this war the Jarman-Abel apparatus, with various other types, were adopted by users in the four services.

With most acute abdomens, chiefly obstructions where a continuous sterile water and glucose infusion has been started some time before the operation, the question of putting another needle into the vein does not arise. It is the practice in such cases to give the patient his usual dose of omnopon and scopolamine, and to inject 4 or 5 c.c. of pentothal into the tube through which flows the sterile water and glucose. To ensure the drug getting into the system successfully, the tube is pinched above the site of the needle puncture and held for a minute or two. The patient goes to sleep and is moved to the operating theatre and the anaesthesia is carried on with a nitrous oxide and oxygen or an oxygen feed. If further relaxation is required or if the patient is becoming resistant, small doses of pentothal may be given repeatedly into the tube whilst the operation is proceeding.
This is necessary when the surgeon requires to get inside the abdomen, whilst doing a laparotomy, and at the time when he wishes to close the abdomen. This type of anaesthetic has been a great success in a large number of cases of obstruction in adults ranging from eighteen to eighty-six, and after other precautions having been taken, leaves little to be desired.

Pentothal supplemented with nitrous oxide and oxygen is all that is needed for all operations outside the abdomen, and generally suffices for an appendicectomy or a colostomy or pelvic operation. It has been my practice (Jarman and Abel, I936) to give pentothal as a means of induction-before giving a spinal-using Etherington Wilson's technique, with a solution of percaine $I: I_{500}$, and supplemented with nitrous oxide and oxygen, for all major abdominal cases such as gastrectomy, cholecystectomy and abdomino-perineal excision of the rectum. The great advantage here is that the patient is entirely unconscious of what kind of anaesthetic has been given-falling asleep in his bed following the intravenous injection. Even in such cases as gastrectomy and cholecystectomy, intermittent pentothal alone has been given, but for the abdomino-perineal operation it has been found wiser to give a spinal anaesthetic.

\section{Effects}

During the induction period the following signs have been noticed:-

Heart.-All cardiac cases have reacted well with the administration of this drug.

Respirations.-These remain steady and gradually become shallow as the drug takes effect. When a full dose has been given the respiratory excursion may become imperceptible. It is wise at this time to give the patient a little oxygen, either by a direct feed or by means of a mask, using a McKesson. To ensure safety, an airway should be inserted as soon as the jaw is relaxed.

Colour.-The patient remains a good colour throughout the operation, but if the respirations become so shallow that the oxygen intake is insufficient, then a slight cyanosis may develop.

Pulse.-The pulse rate at the commencement of the induction remains steady, gradually increasing later. The volume diminishes slightly, but returns after a few minutes.

Eyes.-The eyes behave in much the same way as under nitrous oxide anaesthesia; that is to say, the eyes diverge, the pupils dilate a little and then become central and fixed, the pupil being slightly smaller than normal. Where omnopon and scopolamine have been administered there is no change 
in the size of the pupil due to the previous effect of these drugs. The corneal reflex disappears quite early just before the eyes become central after diverging.

Tremors.-Tremors develop only when the patient is over-stimulated before the drug has had time to take its full effect.

Hiccough.-This symptom usually develops in sensitive people, much more so when the drug is given too quickly. It can be cured quite easily by giving a little $\mathrm{CO}_{2}$ by the direct method.

Sulphonamides.-Where patients have had a course of sulphonamides, and especially M \& B 693, no unpleasant symptoms have developed. It has been reported by various members of the medical profession that barbiturates containing the sulphur atom should not be given with these drugs. I have not noticed any of the severe thromboses of the veins which have been complained of, nor has a serious degree of cyanosis been observed, provided small doses of pentothal are given intermittently. Radley Smith (1940) in his paper written in conjunction with Mallinson, who was his anaesthetist, proved on a large number of military casualties that M \& B 693 could be given before, during, or after the operation, when pentothal had been used, without any unpleasant results. It is firmly believed that thrombosis only develops in such cases where the muscular tone of the vein is lacking or has been damaged. Cyanosis is only observed when an overdose has been given.

Recovery. - The recovery in most cases is uneventful. The majority of patients wake up as though out of a normal sleep. The striking part is that they all ask when the operation is likely to start, not realising that it has been completed and that they are back in bed. About an hour later they have an intense desire to go to sleep again, which they do for probably an hour or more, when they wake up again as though nothing had happened. There has been no vomiting, neither headaches nor chest complications have been noticed, and restlessness occurs only in a very small percentage of cases.

Dangers.-The most obvious danger is an obstructed airway. A Phillip's or Guedel's airway should therefore be inserted whilst the patient is under the anaesthetic. If this is not done, or should the airway be removed too soon, the jaw drops and the base of the tongue falls back and causes an obstruction. To counteract this it is as well either to insert an airway or to open the mouth by means of a gag, apply the tongue forceps to the middle third of the tongue, and at the same time push the lower jaw forwards. Stridor is likely to develop if the patient has some mild form of catarrh or an extremely dirty tongue,,$\vec{\omega}$ and in all these cases the airway should be watched very carefully. An apparatus to administer oxygen $\mathrm{n}_{\mathbb{\mathrm { O }}}$ under pressure should be at hand, since by the administration of this gas the symptom is relieved.

Dosage.-After anaesthesia has been induced $\stackrel{\vec{F}}{\overrightarrow{5}}$ with the initial dose of 5 to Io c.c. of the 5 per cent? solution, 2 or 3 c.c. may be repeated according to $\frac{\bar{\sigma}}{\overline{7}}$ the length of the operation, the relaxation required $\frac{\vec{m}}{\sigma}$ by the surgeon, and in order to keep the patiento from reacting to painful stimuli.

Over-dosage.-This is a danger which unfortu- $-\overrightarrow{0}$ nately occurs far too often. It is not sufficiently $\vec{\omega}$ realised that only part of the maximum dose $\omega$ should be given and not all of it. To overcomę respirator depression artificial respiration will have 3 . to be resorted to as well as the administration of oxygen and carbon dioxide, either by means of $a_{i}^{N}$ mask or by passing of an intratracheal tube. Whilst N this is being done, coramine or picrotoxin should be $\omega$ given intravenously or intramuscularly.

Trouble with the veins. - In the early days, when $\stackrel{\text { ? }}{-}$ pentothal was used in ro per cent solution, an $\vec{O}$ extravenous injection often resulted in the development of a sore. Now that the 5 per cent solu-:0 tion is used, this danger is largely overcome, and $\underline{\mathrm{T}}$ in America where many anaesthetists use a 2 p8r $\overrightarrow{0}$ cent solution this condition seldom arises. It Gs always wise, therefore, to make sure that the blowi flows back into the syringe and, when injecting, see that the needle is kept steady. If there is the slightest sign of swelling round the site of the puncture the plunger should be withdrawn again to $\frac{\partial}{\mathrm{D}}$ see if the blood flows equally well into the syringe. $\frac{2}{2}$ If this is not so, the needle should be removed and $\overrightarrow{0}$ another vein chosen.

There are times when some of the fluid does leak round the vein, and this should be treated at once with a compress of hypertonic saline which should윽 be left on the arm for twenty-four hours. Another method is to treat it with hot fomentations or hot 3 . antiphlogistine.

Injection into an artery. -This unforeseen circumstance has happened on a small number of 9 occasions. The author has had no experience of this. It follows an injection on to the antecubital? space. This can be avoided by noticing the colour of the blood, venous as compared with afterial,. and the resistance to the needle when injecting an 0 artery as compared to a vein. The palpation, colour and position of the vein is usually sufficiento indication to avoid this very unpleasant mistake.

The patient usually complains of pain in his $\mathbb{D}$ forearm and fingers if the artery is pierced, and the ${ }^{?}+$ needle should be withdrawn immédiately. 


\section{Advantages and Disadvantages}

The advantages of this drug are as follows:-

(a) Except for a slight prick in the arm, the patient is unaware of having an anaesthetic.

(b) There is no mask over the face.

(c) There is no sudden concentration of gas or vapour.

(d) The patient is unconscious of being taken to the theatre, or of any subsequent manipulation by the anaesthetist or the surgeon.

(e) On waking the patient does not realise the operation has taken place.

(f) He wakes as if out of a natural sleep.

(g) There is no sickness, vomiting or even headache.

(h) There should be no smell of ether at all in the room, and the patient is usually most grateful for not having had his system saturated with such a drug.

The disadvantages are fere.

(a) Shallow respirations-these have been described and how to overcome them.

(b) The fact that the injection is irreversible. This is an anxiety which is relieved by knowing that the drug is broken down quickly in the blood stream and that as long as the patient is a good colour, and the pulse rate within normal limits, return to consciousness will occur in a short time.

(c) The possibility of not finding the vein and not having another apparatus to carry on the anaesthetic.

\section{Antidotes}

Coramine in large doses from 5 to $\mathrm{I}_{5}$ c.c. is the drug of choice in cases of dire necessity. Picrotoxin, another drug which has been used a great deal in cases of severe barbiturate poisoning, has its followers and can be strongly recommended. 2 to 3 c.c. of 0.3 per cent solution is injected intravenously at intervals of fifteen to twenty minutes.

The antidotes do not differ from those of any other form of general anaesthetic. It is hardly worth pointing out that the McKesson or any such other apparatus, with which oxygen can be given under pressure, also carbon dioxide, should be at hand. Many people also use the drug alphalobeline gr. $\frac{3}{20}$ ths or $\frac{3}{10}$ ths.

\section{Clinical Experiences}

Since I930 my experience now goes to well over 45,000 cases. These of course include a large number using the drug sodium evipan and those closely allied to it. It is still my belief as the result of experience that sodium pentothal is the drug of choice and will remain so until something at least
20 per cent better is produced. All types of cases have been included, from dental cases and minor operations to the various grades of major operations. It has been suggested that a short list of cases should be given where there is no need to use anything else but. pentothal. They are as follows:-

Dental cases.-Straightforward extractions, preparing and filling of cavities.

Bone operations.- Setting of fractures, plasters and removal of sequestra.

Minor gynaecological and genito-urinary operations.

Minor operations. - Removal of external cysts from any part of the body, empyemata, haemorrhoids, fistula and fissures, burns and painful dressings.

Pentothal can also be used in obstetrics but only in small doses. According to reports received, the use of pentothal in obstetrics has been a great success in America, especially for Caesarean section and manipulation of the foetus in utero, but my own experience is small.

The technique of intravenous anaesthesia in children is much the same as in adults after the child has reached the age of fourteen years. Below this age the mere fact of the prick of the needle sometimes intimidates the child. This is overcome by suitable premedication such as nembutal gr. I up to gr. $2 \frac{1}{2}$ according to the age of the patient, given one hour before the operation.

With children up to the age of six, it is wiser and kinder to rely on syrup of chloral dr. $\frac{1}{2}$ to dr. 2 or

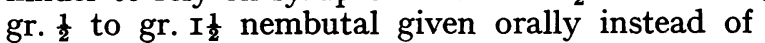
attempting to use the intravenous method. From six to fourteen years, if the child has been properly premedicated, pentothal can be administered without upsetting the patient if skilfully used, the most important points in the technique are a very sharp needle and the location of a vein. It has been noticed that all childen get rid of.the drug rapidly, and on the administration of a second dose they settle down very quickly. It may have a cumulative effect, and in consequence the child needs careful watching until partial consciousness starts to return.

Only in very severe cases have I used continuous pentothal for children where a drip water-glucose infusion has been started some time previous to the operation, and in these cases an extraordinarily small amount of pentothal has been proved necessary. In the majority of cases small doses have been given intermittently, followed by nitrous oxide and oxygen.

It is worth while at this point to describe the experience gained with regard to war wounds.

In France pentothal was used in large quantities by itself to enable the medical officer in charge to do what he could in the front line and, during the retreat, he was able to put the patient into an 
ambulance or lorry whilst in a somnolent state, and get him back to the coast with the minimum amount of pain and discomfort.

In cases suffering from bombs or burns, especially the latter, it had been the practice to give gr. $\frac{1}{4}$ to gr. $\frac{1}{2}$ morphia. When admitted to the theatre the patient was given atropin gr. $\frac{1}{100}$ th, anti-tentanus serum having been administered in the wards previously. A small dose of pentothal, say 3 to 5 c.c., was given into a suitable vein, sometimes in the leg, if the arm had been severely burned. As soon as the patient was asleep the clothing, etc., could be removed, the patient cleaned up and, if necessary, a few more c.cs. given whilst the affected area was treated. Shock seemed to be diminished in all these cases. This was strikingly so in all severe wounds caused by bomb splinters or masonry. In more serious wounds pentothal was used for induction and the anaesthetic was continued with nitrous oxide and oxygen. The blood pressure was taken before and after the operation and whilst recovering in bed. When it started to drop, plasma was given in case of ordinary shock, and this had a most beneficial effect. In other cases, where the patient was severly shocked owing to wounds or to haemorrhage, a saline and glucose drip was started in the ward, which could be changed to whole blood or plasma according to the reading of the blood pressure or the condition of the patient.

Chest complications were negligible, and the reduction of post-operative shock was particularly remarkable. It was felt that this was entirely due to the barbiturate.

Research work has been carried out on the possibility of shock being entirely eliminated by the use of pentothal. It has been proved without doubt that this form of anaesthesia has enabled innumerable operations to be performed that might have been impossible under other circumstances. The convalescent period has been reduced, and mental and physical shock are fast disappearing. It is sufficient to add that the general impression gained by surgeons, anaesthetists and patients, is that intravenous anaesthesia is the method of choice, combined with, or without, other agents. The results with war wounds, bombing and especially severe burns have been more than gratifying when using intravenous anaesthesia.

Another drug has come to light and has gone through the capable hands of research workers in the United States, Canada, Liverpool, and London. This is curare. It is still in its infancy, but is growing up fast.

There is no doubt that this drug, either as tubocurarine chloride intocostrin, or as the synthetic preparation with only a number to distinguish it, certainly produces muscular relaxation when combined with small doses of pentothal. $\overrightarrow{0}$ This drug, like all other new drugs, must be used $\cong$ with care. It is advisable for anasthetists intend- 3 ing to use this new agent, or any other for that $\stackrel{\mathbb{Q}}{2}$ matter, to try and see somebody who has had per-.. sonal experience, so that they can be told what to $\overrightarrow{\vec{c}}$ do in case trouble is encountered.

\section{Results and Conclusion}

The results have been most gratifying, and out of the large number of cases which have been given क barbiturates there have been no fatalities. The $\overrightarrow{0}$ patients are quite willing to have another opera- $\vec{\overrightarrow{ }}$ tion, providing the same type of anaesthetic is re- $\vec{\sigma}$ peated. There has been no serious case of vomiting and often where there has been slight nausea $\frac{0}{3}$ this has probably been due to the effect of the omnopon and scopolamine and it is quite true to say that the proportion of this type of case is considerably less than with any other anaesthetic. $\stackrel{N}{\omega}$ In conclusion, it is my firm belief that pentothal is the anaesthetic of choice, and is an extremely 0 useful addition to the surgical armamentarium provided reasonable precautions are taken. Fol- $\vec{O}$ lowing in the experiences of other anaesthetists as? well as myself, it is considered that the inter-0 mittent method is the one of choice, and that repeat doses should never exceed 2 or 3 c.c. at any opeco time unless under very unusual circumstances. The drug should never be used single-handed, unle⿳⺈冂大冖s conditions specially demand it. It is safer to haveo someone supporting the jaw whilst the anaesthetic is being administered. The patient's airway shouldo be continuously maintained. Precautions as for any other anaesthetic should always be at hand namely, an apparatus like the McKesson, Magill's] tubes, a suction apparatus, and all the necessary cardiac and respiratory stimulants.

As long as it is recognised that theinduction period. is as quick, if not quicker, than with nitrous oxide there should be little danger in teaching students? how to use pentothal, providing they have a goods grounding in dental anaesthetics, and intravenous technique.

\section{REFERENCES}

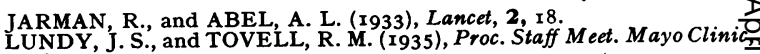

10,257, I935.
JARMAN, R., and ABEL, A. L. (1936), "Pentothal Sodium, Intravenoußs Anaesthesia," Lancet, 1, 42

QUAYLE, G. (r940), "Continuous Intravenous Anaesthesia for Gynaeco-

JARMAN, R. (1936), "Modern Anaesthesia," Proc. Grad. Med. Jour., 1 ho

RADLEY SMITH, E. J. (1940), “A Sulphur Compound in Conjunction

with Sulphapyridine," Brit. Med. J., 2, 488.

PRESCOTT, ORGANE and ROWBOTHAM (1946), Lancet, July 20 t

DOUG6, 6412.

CAMERON and SARUM, J. of Path. and Bacteriol., Vol. XLVIII, 1939, p. 49 . 